\title{
Anti-obesity effect in high-fat-diet-induced obese C57BL/6 mice: Study of a novel extract from mulberry (Morus alba) leaves fermented with Cordyceps militaris
}

\author{
MI RIM LEE ${ }^{1}$, JI EUN KIM ${ }^{1}$, JUN YOUNG CHOI ${ }^{1}$, JIN JU PARK ${ }^{1}$, HYE RYEONG KIM ${ }^{1}$, BO RAM SONG ${ }^{1}$, \\ YOUNG WHAN CHOI ${ }^{2}$, KYUNG MI KIM ${ }^{3}, \mathrm{HYUNKEUN} \mathrm{SONG}^{4}$ and DAE YOUN HWANG ${ }^{1}$ \\ Departments of ${ }^{1}$ Biomaterials Science and ${ }^{2}$ Horticultural Bioscience, College of Natural Resources and \\ Life Science/Life and Industry Convergence Research Institute, Pusan National University, Miryang, \\ Gyeongsangnam 50463; ${ }^{3}$ Life Science Research Institute, Novarex Co., Ltd., Chungju, Chungcheong 28126; \\ ${ }^{4}$ Biomedical Science Institute, Changwon National University, Changwon, Gyeongsangnam 51140, Republic of Korea
}

Received January 16, 2018; Accepted August 14, 2018

DOI: $10.3892 / \mathrm{etm} .2019 .7191$

\begin{abstract}
The therapeutic effects of mulberry (Morus alba) leaves on lipid metabolism, including lipogenesis, lipolysis and hyperlipidemia are widely known, although their fermented products are yet to be applied. To investigate the therapeutic effects of a novel extract of mulberry leaves fermented with Cordyceps militaris (EMfC) on lipid metabolism, the lipid profile of serum, lipid accumulation, lipolytic activity and lipogenesis regulation were measured in high fat diet (HFD)-induced obese C57BL/6 mice treated with EMfC for 12 weeks. Briefly, the concentrations of low-density lipoprotein, triglyceride, total cholesterol and glucose significantly decreased in the serum of the HFD+EMfC treated group when compared with the HFD+Vehicle treated group, while the levels of high-density lipoprotein increased in the HFD+EMfC group. The amount of abdominal fat and the size of adipocytes were significantly lower in the HFD+EMfC treated group when compared with the HFD+Vehicle treated group. The weight and number of lipid droplets of liver tissue exhibited
\end{abstract}

Correspondence to: Professor Dae Youn Hwang, Department of Biomaterials Science, College of Natural Resources and Life Science/Life and Industry Convergence Research Institute, Pusan National University, 50 Cheonghak-ri, Samnangjin, Miryang, Gyeongsangnam 50463, Republic of Korea

E-mail: dyhwang@pusan.ac.kr

Professor Hyunkeun Song, Biomedical Science Institute, Changwon National University, 20 Changwondae-ro Uichang, Changwon, Gyeongsangnam 51140, Republic of Korea

E-mail:kali71@hanmail.net

Abbreviations: EMfC, extract of mulberry leaves fermented with Cordyceps militaris; HFD, high fat diet; TG, triglyceride; TC, total cholesterol

Key words: obesity, mulberry leaves, fermentation, Cordyceps militaris, lipolysis, lipogenesis a similar decrease. Furthermore, the mRNA levels of peroxisome proliferator-activated receptor- $\gamma$ for adipogenesis as well as adipocyte protein 2 and Fas cell surface death receptor for lipogenesis reduced following EMfC treatment for 12 weeks. Phosphorylation of perilipin and hormone-sensitive lipase, and in the adipose triglyceride lipase expression showed a significant increase in the HFD+EMfC treated group. These results indicated that EMfC may prevent fat accumulation in the HFD-induced obese C57BL/6 mice through the inhibition of lipogenesis and by stimulating lipolysis. Thus, the results provide evidence for the potential use of EMfC as an anti-obesity complex in the treatment of obesity.

\section{Introduction}

Globally, obesity is a serious health problem due to its strong association with increased dyslipidemia, cardiovascular disease (including hypertension, stroke, myocardial infarction), insulin resistance, glucose metabolism disorders, osteoarthritis and some cancers $(1,2)$. According to a recent report, the increase in the world obesity rate from 1975 to 2014 was $3.2-10.8 \%$ in men, and $6.4-14.4 \%$ in women (3). This trend will have a significant impact on world health and the economy. The main causes of obesity are the delicious and energetic diets rich in fat, consumption imbalances, low momentum, fat, sugar and salt (1). The most common methods for preventing and treating obesity are maintaining an optimal weight by calibrating lifestyle habits by introducing well-structured calorie restriction and exercise programs (4). Lifestyle corrections are very challenging for obese patients, who subsequently resort to the use of medications as an adjunct. However, factors such as the cost and potential side effects are the main causes for failure of weight and obesity management with medication. Recent reports indicate that many herbal extracts, including Paullinia cupana, Plantago psyllium, Garcinia cambogia, and Ilex paraguariensis, are relatively safe for controlling weight, by promoting the lipid and carbohydrate metabolism (5). Thus, herbal extracts from natural sources are likely to be safe and effective alternatives for treating obesity. 
Commonly known as mulberry in Korea, Morus alba (family: Mulberry), grows in northern China, and has been cultivated from India to the Middle East, southern Europe, and recently North America. Pharmacological studies have reported the roots and bark to have antibacterial (6), antioxidant and hypoglycemic (7,8), and neuroprotective, anti-ulcer, analgesic and anti-inflammatory activities (9-12). Several studies have also presented the beneficial effects of mulberry leaves on lipid metabolism. Kobayashi et al (13) reported that administration of mulberry leaves to rats for 7 weeks induced the upregulation of fatty acid oxidation and downregulation of lipogenesis-related genes. Other studies reported that the mulberry leaf extract significantly reduced the lipid accumulation and lipoprotein-related protein expression in 3T3-L1 adipocytes (14). Additionally, the mulberry leaf extract stimulates the glucose uptake in rat adipocytes, and reduces the adipocyte development in white adipose tissue extracted from $\mathrm{db} / \mathrm{db}$ mice through inhibition of oxidative stress (15-17). Cordyceps militaris (C. militaris) is an insect pathogen belonging to Ascomycota, and is traditionally used in Chinese and East Asian medicine $(18,19)$. Cordycepin isolated from C. militaris is effective in anticancer (20), antitumor (21), antimicrobial (22) and antioxidant (23) therapy. Recent reports indicate that C. militaris-fermented products are beneficial in attenuating liver fibrosis $(24,25)$; similar effects were observed with a mixture of C. militaris and a secondary metabolite secreted from mushroom (26). In our previous study, we reported that the extract of Morus alba leaves fermented with C.militaris (EMfC) has a lipolytic effect on adipocytes isolated from SD rats (27). However, no detailed study was performed to evaluate the inhibitory effect of EMfC on obesity.

Based on previous reports, we hypothesized that the EMfC induces its anti-obesity effects through the regulation of lipid metabolism. We therefore used mice with high-fat diet-induced obesity to evaluate changes in fat accumulation and lipid profiles, as well as examined the underlying mechanisms of adipogenesis, lipogenesis and lipolysis.

\section{Materials and methods}

Preparation of EMfCs. One of the authors (Professor Young Whan Choi) characterized the samples of mulberry leaves collected from plantations in the Sangju district of Korea in October 2015. Voucher specimens (accession no. Mul-PDRL-1) were deposited in the herbarium of the Pusan National University (Miryang, Korea). Professor Sang Mong Lee of the Department of Life Science and Environmental Biochemistry, Pusan National University, kindly provided the $C$. militaris used for fermentation. The Jeongeup Agriculture Cooperative Federations for Silkworm Farming (Jeongeup, Korea) supplied the silkworm pupae powder.

Briefly, the mulberry leaves were powder using an electric blender, after drying in a hot-air drying machine (JSR, Seoul, Korea) for $24 \mathrm{~h}$ at $60^{\circ} \mathrm{C}$. The mulberry powder was sterilized by autoclaving at $121^{\circ} \mathrm{C}$ for $60 \mathrm{~min}$, mixed with $50 \%$ silkworm powder (SWP), and the mixture inoculated with $10 \%$ C. military $(\mathrm{v} / \mathrm{w})$. The samples were incubated in a shaking incubator (cat. no. SI-600R; Lab Companion, Seoul, Korea) at $150 \mathrm{rpm}$ and $25^{\circ} \mathrm{C}$, and fermented for 4 weeks. On the last day of fermentation, the mixture was harvested from the flask and extracted with $95 \% \mathrm{EtOH}$. To prepare the extracts of fermented mulberry powder, the extracted powder was mixed with the solvent $(95 \% \mathrm{EtOH})$ in a fixed liquid ratio (mulberry powder:solvent, ratio 1:10) and sonicated for $1 \mathrm{~h}$ using a JAC ultrasonic device (KODO, Hwangseong, Korea). Centrifuging at 3,000 rpm for $10 \mathrm{~min}$ separated the supernatant from the sonicated extract. The pellet was resuspended in $9 \mathrm{ml}$ of the solvent, and further sonicated using the same conditions. This procedure was repeated once more, and the resultant supernatant was collected, filtered through a $0.4 \mu \mathrm{m}$ filter (cat. no. HAWP04700; Millex-LH, MerckMillipore, Darmstadt, Germany), and evaporated using a vacuum evaporator (cat. no. R-300, BUCHI Corporation, New Castle, Delaware, USA). Finally, lyophilization of the EMfC was achieved using the circulating extraction equipment (IKA Labortechnik, Staufen, Germany). The extracts were dissolved in DMSO (cat. no. D2660, Sigma-Aldrich Co., St Louis, MO, USA) at a concentration of $50 \mathrm{mg} / \mathrm{ml}$ before use.

Design of animal experiment. Eight-week-old C57BL/6 male mice were purchased from Samtako Bio-Korea Inc. (Osan, Korea), and provided ad libitum access to water and a standard irradiated chow diet (Samtako Bio-Korea Inc.). During the experiment, mice were maintained in a pathogen-free state under a strict light cycle (lights on at 08:00 h and off at 20:00 h) at $23 \pm 2^{\circ} \mathrm{C}$ and $50 \pm 10 \%$ relative humidity. All C57BL/6 mice were handled at the Pusan National University-Laboratory Animal Resources Center, accredited by the Korea Food and Drug Administration (FDA; Accredited Unit no. 000231) and AAALAC International (Accredited Unit no. 001525).

The Pusan National University Animal Ethics Committee (PNU-2017-1519) approved the protocol for animal study. All animals were acclimatized on a normal diet (D12450K; Research Diets, New Brunswick, NJ, USA) for 1 week. The C57BL/6 mice were then divided into 4 study groups (7 mice/group): (1) Control group fed a normal diet (No treatment group), (2) group fed HFD (high fat diet) plus vehicle (olive oil+1\% DMSO) (HFD+Vehicle treated group), (3) group fed HFD plus $10 \mathrm{mg} / \mathrm{kg}$ Orlistat (Sigma-Aldrich Co.) (HFD+OT treated group), and (4) group fed HFD plus $50 \mathrm{mg} /$ $\mathrm{kg}$ EMfC (HFD+EMfC treated group). For 12 weeks, mice of all HFD treatment groups consumed HFD containing $60 \%$ kcal fat purchased from Research Diets (cat. no. D12492; Research Diets, Inc., New Brunswick, USA). After $24 \mathrm{~h}$ of the final EMfC treatment, all mice were euthanized using $\mathrm{CO}_{2}$ gas, after which the tissue samples were acquired and stored in Eppendorf tubes at $-70^{\circ} \mathrm{C}$ until assay.

Measurement of body weight, dietary intake and organ weight. Throughout the experimental period, the body weight of mice treated with Vehicle, OT or EMfC were measured daily at 10:00 am using an electronic balance (cat. no. AD-2.5; Mettler Toledo, Greifensee, Switzerland) according to the KFDA guidelines. In addition, the weights of liver and abdominal organs collected from the sacrificed C57BL/6 mice were determined using the same method employed to measure the body weight. Feed intake was measured at the same time once a week during the study period, using a chemical balance (Mettler Toledo, Switzerland). 
Serum biochemical analysis. After 12 weeks of feeding experimental diets, blood was collected from the abdominal veins of all C57BL/6 mice after fasting for $18 \mathrm{~h}$. The blood samples were incubated for $30 \mathrm{~min}$ at room temperature in serum separating tubes (BD Biosciences, Franklin Lakes, NJ, USA). Serum was obtained by centrifugation at $1,500 \mathrm{xg}$ for $15 \mathrm{~min}$. Serum glucose, TC (total cholesterol), TG (triglyceride), high-density lipoprotein (HDL)-cholesterol, and low-density lipoprotein (LDL)-cholesterol levels were analyzed by the automatic chemical analyzer (BS-120 Chemistry Analyzer; Mindray, Shenzhen China). Additionally, serum was analyzed for alkaline phosphatase (ALP), alanine aminotransferase (ALT), aspartate aminotransferase (AST), blood urea nitrogen (BUN), and creatinine (Crea) by using an automatic biochemical analyzer (BS-120; Mindray). All assays were conducted in duplicate using fresh serum.

Histopathological analysis. Liver and fat tissues dissected from mice of all subset groups were fixed in $10 \%$ neutral buffered formaldehyde ( $\mathrm{pH}$ 6.8) overnight. The dehydrated liver tissue was then embedded in paraffin wax. Next, a series of liver and fat sections $(4 \mu \mathrm{m})$ were cut from the paraffin-embedded tissues using a Leica microtome (cat. no. DM500; Leica Microsystems, Bannockburn, IL, USA). These sections were then deparaffinized with xylene (cat. no. 8587-4410; Daejung, Gyeonggi-do, Korea), rehydrated with graded ethanol (decreasing concentrations of 100-70\%), and finally washed with distilled water. The slides with liver sections were stained with hematoxylin (cat. no. MHS16) and eosin (cat. no. HT110332; both Sigma-Aldrich Co.), washed with $\mathrm{dH}_{2} \mathrm{O}$, and pathological changes were assessed using the Leica Application Suite (Leica Microsystems).

Reverse transcription-quantitative polymerase chain reaction $(R T-q P C R)$ analysis. Frozen liver tissue was chopped with scissors and homogenized in RNA Bee solution (cat. no. CS-105B; Tet-Test Inc., Friendswood, TX, USA). Total RNA molecules were isolated by centrifugation at $15,000 \mathrm{rpm}$ for $15 \mathrm{~min}$, after which the concentration was measured by UV spectroscopy. The complementary DNA (cDNA) was synthesized by Invitrogen Superscript II reverse transcriptase (cat. no. 4376600; Thermo Scientific, Wilmington, Delaware, USA). Quantitative PCR was performed with the cDNA template $(2 \mu \mathrm{l})$ and $2 \mathrm{X}$ Power SYBR-Green $(6 \mu \mathrm{l}$; Toyobo Life Science, Osaka, Japan) containing specific primers. The primer sequences for target gene expression identification used were as follows: PPAR $\gamma$ sense, 5'-GAGTTCATGCTTGTG AAGGATGCAAGG-3' and anti-sense, 5'-CATACTCTGTGA TCTCTTGCACG-3'; C/EBP $\alpha$ sense, 5'-GTGGACAAGAAC AGCAACGAGTACC-3' and anti-sense, 5'-GGAATCTCC TAGTCCTGGCTTGC-3'; $\beta$-actin sense, 5 '-TGGAATCCT GTGGCATCCATGAAAC-3' and anti-sense, 5'-TAAAAC GCAGCTCAGTAACAGTCCG-3'. qPCR was performed for 40 cycles using the following parameters: Denaturation at $95^{\circ} \mathrm{C}$ for $15 \mathrm{sec}$, followed by annealing and extension at $70^{\circ} \mathrm{C}$ for $60 \mathrm{sec}$. Fluorescence intensity was measured at the end of the extension phase of each cycle. The threshold value for the fluorescence intensities of all samples was set manually. The reaction cycle at which the PCR products exceeded this fluorescence intensity threshold during the exponential phase of
PCR amplification was considered as be the threshold cycle (Cq). Expression of the target gene was quantified relative to that of the housekeeping genes $\beta$-actin, based on comparison of the Cqs at a constant fluorescence intensity, as per Livak and Schmittgen's method (28).

Western blot analysis. Liver tissue $(50 \mathrm{mg})$ collected from each group was homogenized using PRO-PREPTM Solution (cat. no. 170841; iNtRON Biotechnology Inc., Sungnam, Korea), and total protein extracts were collected by centrifugation at $13,000 \mathrm{rpm}$ for $5 \mathrm{~min}$. The prepared proteins were subsequently subjected to $10 \%$ SDS-PAGE for $2 \mathrm{~h}$ at $100 \mathrm{~V}$, and transferred to a nitrocellulose membrane (GE Healthcare, Little Chalfont, UK) for $2 \mathrm{~h}$ at $40 \mathrm{~V}$ in transfer buffer $(25 \mathrm{mM}$ Trizma-base, $192 \mathrm{mM}$ glycine, and 20\% methanol). Membranes were then exposed to appropriate dilutions of primary antibodies and allowed to hybridize overnight at $4^{\circ} \mathrm{C}$ : anti-perilipin antibody (cat. no. 9349S), anti-p-perilipin antibody (cat. no. 9621), anti-HSL antibody (cat. no. 4107S), anti-p-HSL antibody (cat. no. 4139S), anti-ATGL antibody (cat. no. $2183 \mathrm{~S}$ ) and anti- $\beta$-actin antibodies (cat. no. 4967S; all Cell Signaling Technology, Danvers, MA, USA). After removal of the antibodies, the membranes were washed three times in a $10 \mathrm{mM}$ Trizma-base $(150 \mathrm{mM} \mathrm{NaCl}$ and $0.05 \%$ Tween-20) solution for $10 \mathrm{~min}$. The membranes were subsequently incubated with horseradish peroxidase-conjugated anti-secondary antibody for $1 \mathrm{~h}$ at room temperature, after which they were washed again as described above, and developed using the Enhanced Chemiluminescence Reagent Plus kit (cat. no. DG-WF100; Dogen, Seoul, Korea). Finally, the results were quantified using the Image Analyzer System (Fluorchem FC2; Alpha Innotech, CA, USA) and expressed as the fold-increase over control values.

Statistical significance analysis. One-way analysis of variance was performed using SPSS 10.10 (SPSS, Inc., Chicago, IL, USA) in order to determine the variance and identify significant differences between the treated group and other groups, as well as between the vehicle group and other HFD fed groups. All values are presented as the mean \pm standard deviation. $\mathrm{P}<0.05$ was considered to indicate a statistically significant difference.

\section{Results}

Effect of EMfC on body weight and lipid profile of serum. To evaluate the anti-obesity effects of EMfC, we analyzed the changes in body weight and serum lipid profile of HFD-induced obesity mice treated with EMfC for 12 weeks (Fig. 1). Although no significant differences were detected in the body weight of HFD+Vehicle treated group and HFD+EMfC treated group, the serum lipid profile differed after EMfC treatment. As shown in Fig. 1B, all lipid profile factors were 40-180\% higher in the HFD+Vehicle treated group than in the No treatment group. However, the concentration of LDL, TG, TC and glucose significantly decreased in the HFD+EMfC treated group as compared to the HFD+Vehicle treated group $(\mathrm{P}<0.005$; Fig. 1B). Additionally, EMfC treatment resulted in enhanced HDL levels (Fig. 1B). These results suggest that EMfC induces a recovery of the serum lipid profile in HFD-induced obesity mice, although it is unable to inhibit weight gain due to HFD. 

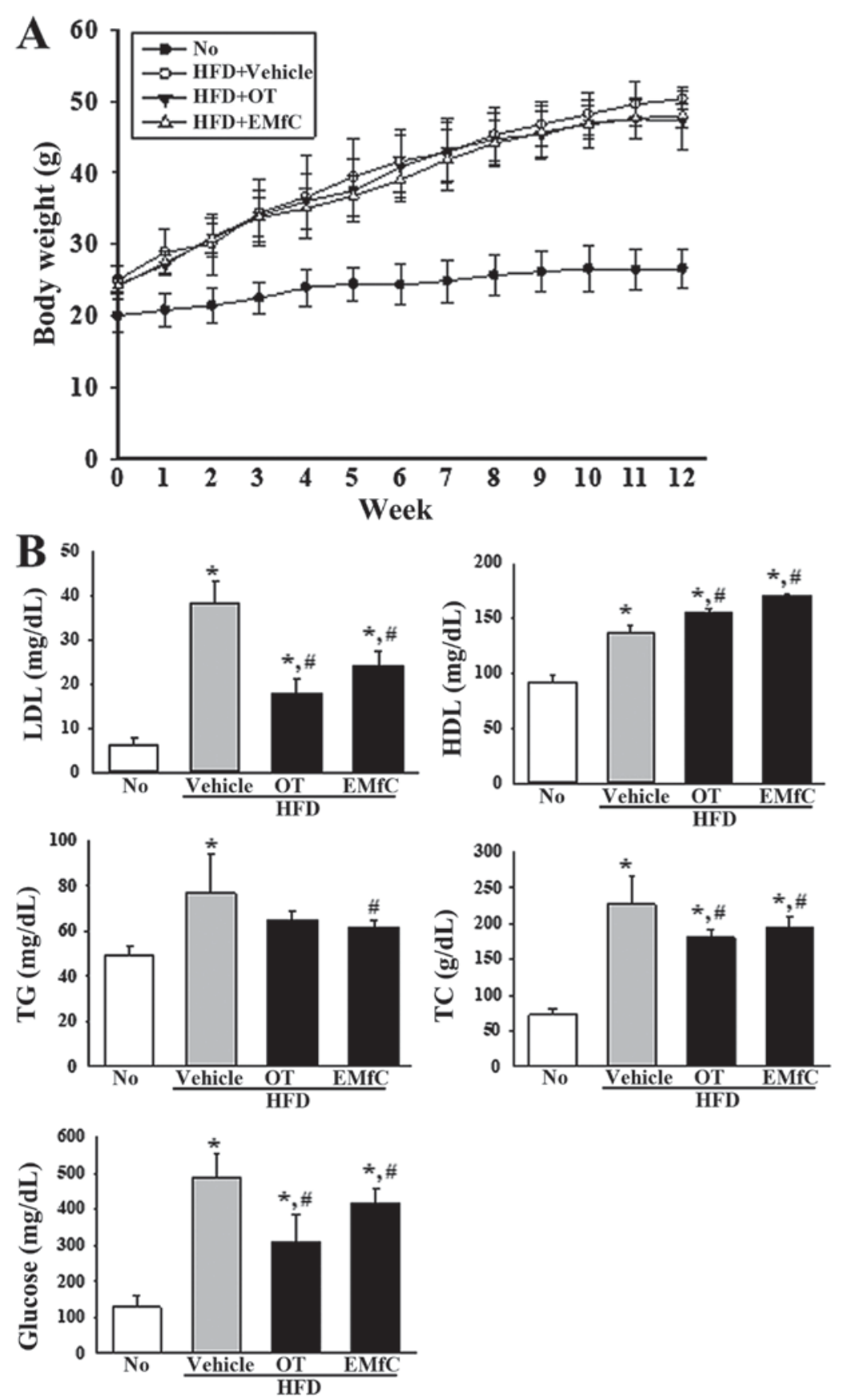

Figure 1. Assessment of the body weight and serum lipid profile. (A) Comparison of body weight following treatment with Vehicle, OT or EMfC in HFD-induced obese C57BL/6 mice. Percentage body weight gain from 0 to 12 weeks. (B) LDL, HDL, TG, TC and glucose content were measured in serum from all mice in the experimental groups. Each group included seven animals. Data are presented as mean percentage weight gain \pm standard deviation. ${ }^{*} \mathrm{P}<0.05$ vs. the No treatment group; ${ }^{~} \mathrm{P}<0.05$ vs. the HFD+Vehicle treated group. LDL, low density lipid; HDL, high density lipid; TG, triglyceride; TC, total cholesterol; OT, Orlistat; HFD, high fat diet; EMfC, extract of mulberry leaves fermented with Cordyceps militaris.

Effect of EMfC on abdominal fat accumulation. In order to investigate the effect of EMfC on abdominal fat accumulation, we assessed the amount of abdominal fat and the size of adipocytes by H\&E staining. The HFD+Vehicle treated group had higher content of abdominal fat as compared to the No treatment group $(\mathrm{P}<0.005)$. However, levels significantly decreased in the HFD+EMfC and HFD+OT treated groups as compared to the HFD+Vehicle treated group $(\mathrm{P}<0.005$; Fig. 2A). A similar pattern was observed for the average area of single adipocyte of fat tissue. HFD+EMfC and HFD+OT treated groups showed significant decrease of the adipocyte area as compared with the HFD+Vehicle treated group $(\mathrm{P}<0.005$; Fig. 2B). These results suggest that EMfC prevents the accumulation of abdominal fat in HFD-induced obesity mice.

Effects of EMfC on hepatic steatosis of HFD-induced obesity mice. Liver weight alterations and hepatic steatosis are common obesity-related phenomena. Therefore, we measured the changes in weight and fat accumulation of liver in HFD-induced obesity mice treated with EMfC. The HFD+Vehicle treated group showed increased liver weight compared to the No treatment 
A
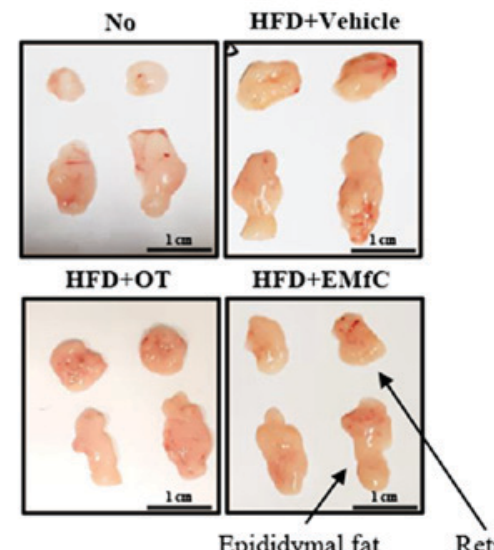

Epididymal fat

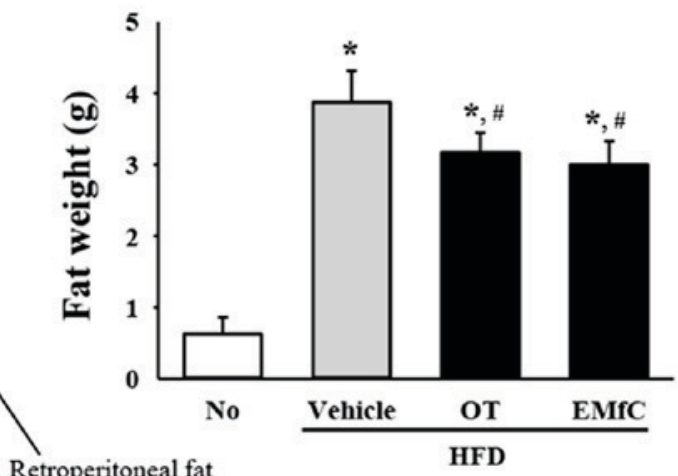

B
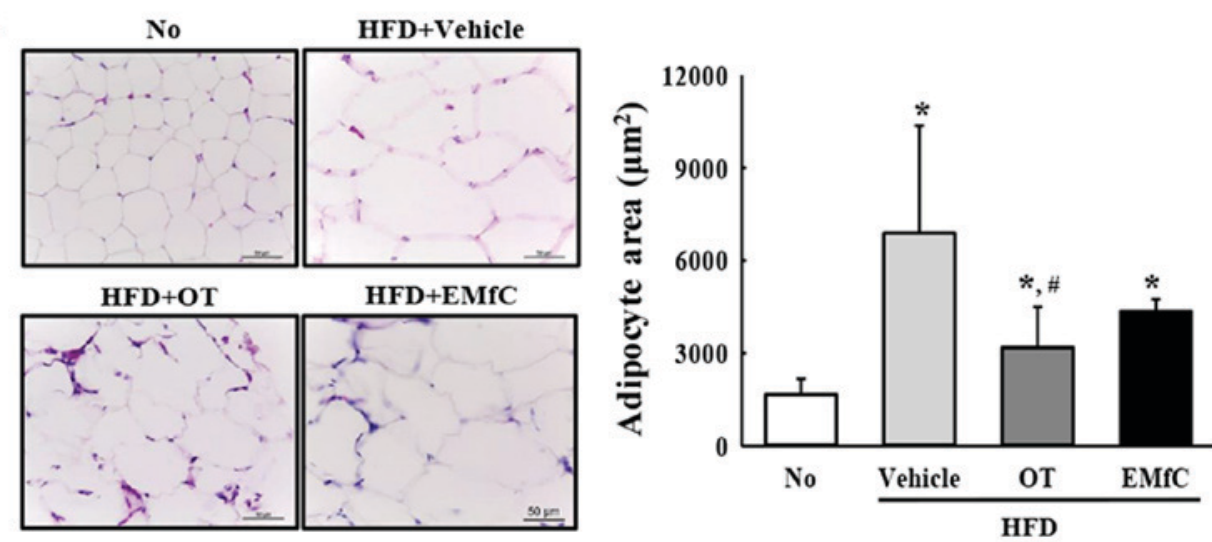

Figure 2. Measurement of fat weight and adipocyte size. (A) Abdominal adipose tissue obtained in the No, HFD+Vehicle, HFD+OT or HFD+EMfC treated group in C57BL/6 mice. Epididymis and retroperitoneal fat was collected from the abdominal region of mice of all subset groups, and their weights were measured using an electrical balance. (B) Hematoxylin and eosin-stained photomicrographs of the epididymal adipose tissue sections (magnification, x200). The rea of adipocyte size is also presented as graphs. Data represent the mean \pm standard deviation from three replicates. ${ }^{*} \mathrm{P}<0.05$ vs. the No treatment group; ${ }^{\#} \mathrm{P}<0.05$ vs. the HFD+Vehicle treated group. HFD, high fat diet; OT, Orlistat; EMfC, extract of mulberry leaves fermented with Cordyceps militaris.

group $(\mathrm{P}<0.005)$. These changes recovered with OT or EMfC treatment (Fig. 3A). Also, significant changes were detected in the number of lipid droplet in the liver tissues of all groups stained with H\&E. The liver section of the HFD+Vehicle treated group showed dramatic increase in the number of lipid drops compared to the No treatment group. In comparison, only a few lipid drops were seen in the HFD+OT and HFD+EMfC treated groups $(\mathrm{P}<0.005$; Fig. 3B). These results indicate that EMfC significantly inhibits the liver hypertrophy, fat accumulation and hepatic steatosis in HFD-induced obesity mice.

Effects of EMfC onadipogenesis and lipogenesis of HFD-induced obese mice. To further elucidate the mechanisms by which EMfC affects obesity, we measured the mRNA levels of several key genes that affecting adipogenesis and lipogenesis in the liver tissue. As shown in Fig. 4, there were significant changes in the mRNA levels of adipogenesis and lipogenesis related genes after EMfC treatment. The mRNA levels of PPAR $\gamma$ and $\mathrm{C} / \mathrm{EBP} \alpha$ for adipogenesis increased after HFD feeding (HFD+Vehicle treated group) compared to the No treatment group $(\mathrm{P}<0.005)$. However, these levels were dramatically decreased after OT or EMfC treatment (HFD+OT and HFD+EMfC treated groups, respectively), although no changes were seen in the $\mathrm{C} / \mathrm{EBP} \alpha$ level (Fig. 4A). The gene expression of aP2 and FAS were showed dramatically increased in the HFD+Vehicle treated group, but significantly decreased in the HFD+OT and HFD+EMfC treated groups when compared to the No treatment group $(\mathrm{P}<0.005$; Fig. 4B). These results indicate that EMfC treatment probably inhibits adipogenesis and lipogenesis in the liver tissue through the regulation of PPAR $\gamma, \mathrm{aP} 2$ and FAS expression.

Effect of EMfC on lipolysis of HFD-induced obese mice. We finally investigated the molecular mechanism for lipolytic activity of EMfC by analyzing the expression and activation of key enzymes involved in triglyceride metabolism. We evaluated the phosphorylation or expression of three major enzymes, including perilipin, HSL and ATGL. We observed increased protein levels of ATGL in the liver tissues after treatment in the HFD+OT and HFD+EMfC groups, as compare to No treatment and HFD+Vehicle treated groups $(\mathrm{P}<0.005$; Fig. 5). The protein levels of perilipin and HSL significantly decreased after OT and EMfC treatment, compared to the HFD+Vehicle treated group. Interestingly, the phosphorylation of perilipin and HSL were significantly enhanced in the HFD+EMfC treated group, while an increase in phosphorylation of perilipin was observed in the HFD+OT treated group $(\mathrm{P}<0.005$; Fig. 5). Our data indicates that increased lipolytic ability of EMfC is mainly dependent on increased expression of ATGL and increased activation of perilipin, as well as increased activation of HSL, unlike OT. 
We therefore suggest that compared to OT, EMfC is likely to be more efficient in contributing to lipolysis.

\section{Discussion}

Recent pharmacological approaches for treating and controlling obesity have resorted to the use of drugs to induce anorexia, inhibit nutritional absorption, and promote weight loss (2). Clinically available anti-obesity agents include pancreatic lipase inhibitors such as orlistat, and appetite suppressants that act on the central nervous system, such as lorcaserin $(29,30)$. Since body fat accumulation is a major risk factor for chronic diseases including hyperlipidemia, diabetes, cardiovascular disease and cancer, interest has increased for developing of new drugs having lipolytic and lipogenic activities $(31,32)$. Numerous studies reported that several lipolytic agents, including norepinephrine, isoproterenol, phoscholine, dibutyl-cAMP (DBcAMP) and theophylline induce lipolysis in adipocytes (33). However, these anti-obesity drugs are associated with serious side effects such as constipation, insomnia, vomiting, headache, abdominal pain, and myocardial infarction (34). Therefore, the development of anti-obesity agents derived from natural products that are effective in weight management in suppressing and decomposing the fat accumulation, and having minimal side effects, are required (35). In this study, to analyze the anti-obesity effect of EMfC, we used orlistat as a positive control for assessing the anti-obesity effect in HFD-induced obese mice. At the end of the 12 weeks study period, although the HFD treated mice had maximum weight gain, there was no effective weight loss with orlistat and EMfC treatments also. This could be because orlistat is an anti-obesity agent involved in fat metabolism rather than weight loss effect. Secondly, the concentration of orlistat used in the study is $10 \mathrm{mg} / \mathrm{kg}$, which is a low concentration as compared to the commonly used concentration of $10-50 \mathrm{mg} / \mathrm{kg}$. The third reason is that although the final effect of orlistat is reduction of body weight following the suppression of fat absorption, 12 weeks is considered the period when no effective weight loss has occurred. Subsequently, we expect similar effects of orlistat and EMfC on fat metabolism, resulting in weight loss during longer study protocols.

In addition, to study the changes in body weight as a consequence of changes in dietary intake (data not shown), a slight decrease was observed in dietary intake of the experimental group (including the vehicle group), with no obvious difference between. We believe this slightly reduction in consumption may be due to the vehicle, and not olistat and EMfC.

Our study data reveals that EMfC treatment inhibits the visceral (epididymal and abdominal) adipose tissue mass, adipocyte cell size, serum LDL, serum TG, serum TC and serum glucose in obese mice induced by HFD. In addition, decreased liver weight and decreased lipid drop after EMfC exposure suggests that EMfC has an anti-obesity effect by being involved in liver lipid metabolism. Interestingly, the serum analysis of the experimental group including EMfC treatment for 12 weeks showed no adverse side effects such as hepatotoxicity and kidney toxicity (data not shown). Taken together these results suggest that the reduction in fat mass observed in HFD+EMfC treated mice is due not to the toxicity

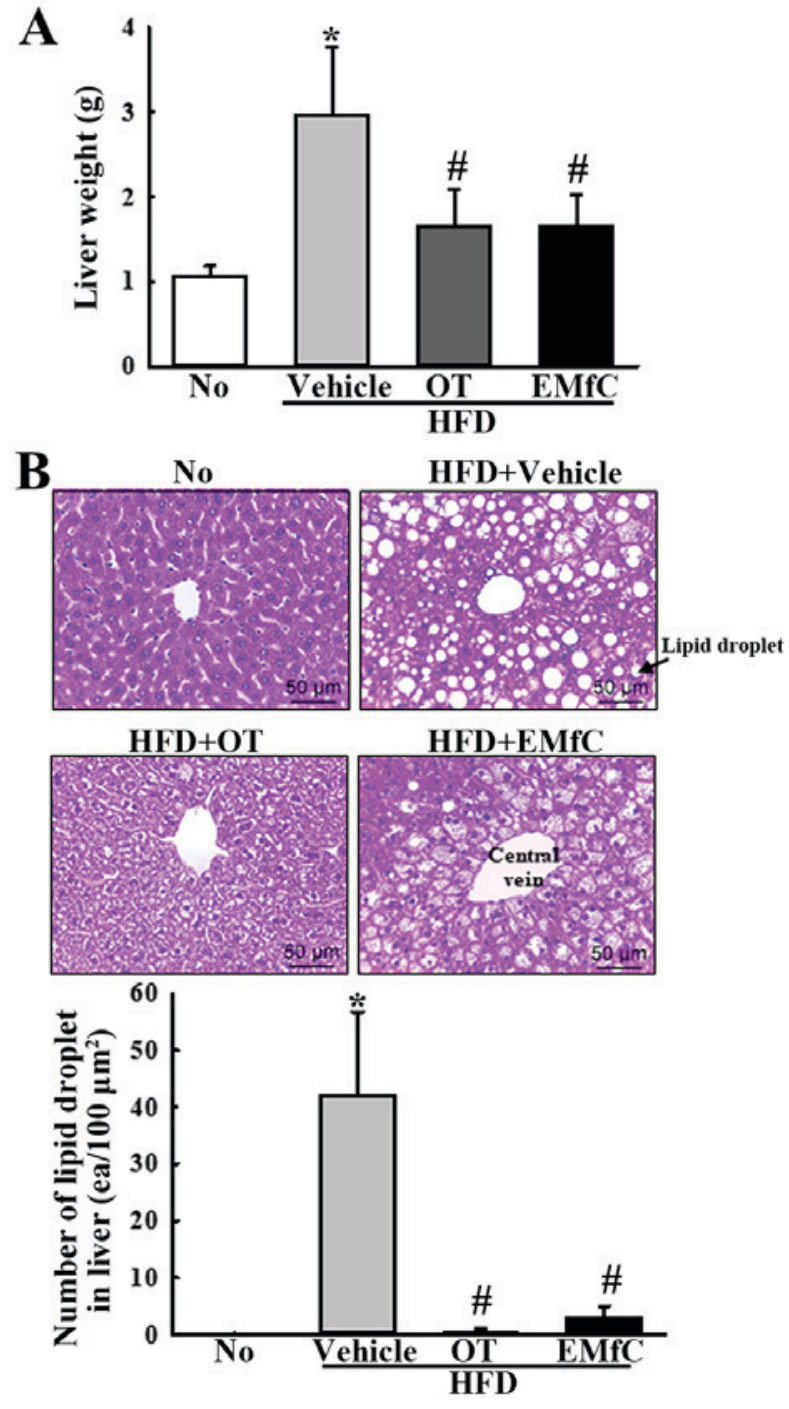

Figure 3. Measurement of liver weight and lipid droplet number of liver. (A) Livers collected from HFD-induced obese C57BL/6 mice following treatment of Vehicle, OT or EMfC, were weighed using an electrical balance. (B) Hematoxylin and eosin-stained photomicrographs of the liver tissue sections (magnification, x200). The number of lipid drops in the liver is presented as a graph. Data represent the mean \pm standard deviation from three replicates. ${ }^{*} \mathrm{P}<0.05$ vs. the No treatment group; ${ }^{\prime} \mathrm{P}<0.05$ vs. the HFD+Vehicle treated group. HFD, high fat diet; OT, Orlistat; EMfC, extract of mulberry leaves fermented with Cordyceps militaris.

of EMfC, but due to the direct pharmacological action on adipose tissue.

EMfC treatment in HFD fed mice decreased the serum TG, TC, LDL and glucose levels proportionate to the decrease in adipose tissue mass. Conversely, the serum HDL concentration dramatically increased after EMfC and OT treatment. triglycerides (TGs) accumulate in adipose tissues by forming lipid drops of adipocytes (36). Our data displayed that the level of TG only marginally after EMfC treatment. Microscopic and histologic examinations revealed reduction in the mass of adipose tissue and adipocyte cell sizes of the HFD+EMfC treated groups as compared to the HFD+Vehicle treated group. The liver weight and number of lipid drops also decreased in the HFD+EMfC treated organ. Although the effect on serum level reduction of accumulated TGs is marginal, EMfC reduces adipose tissue due to decreasing of 

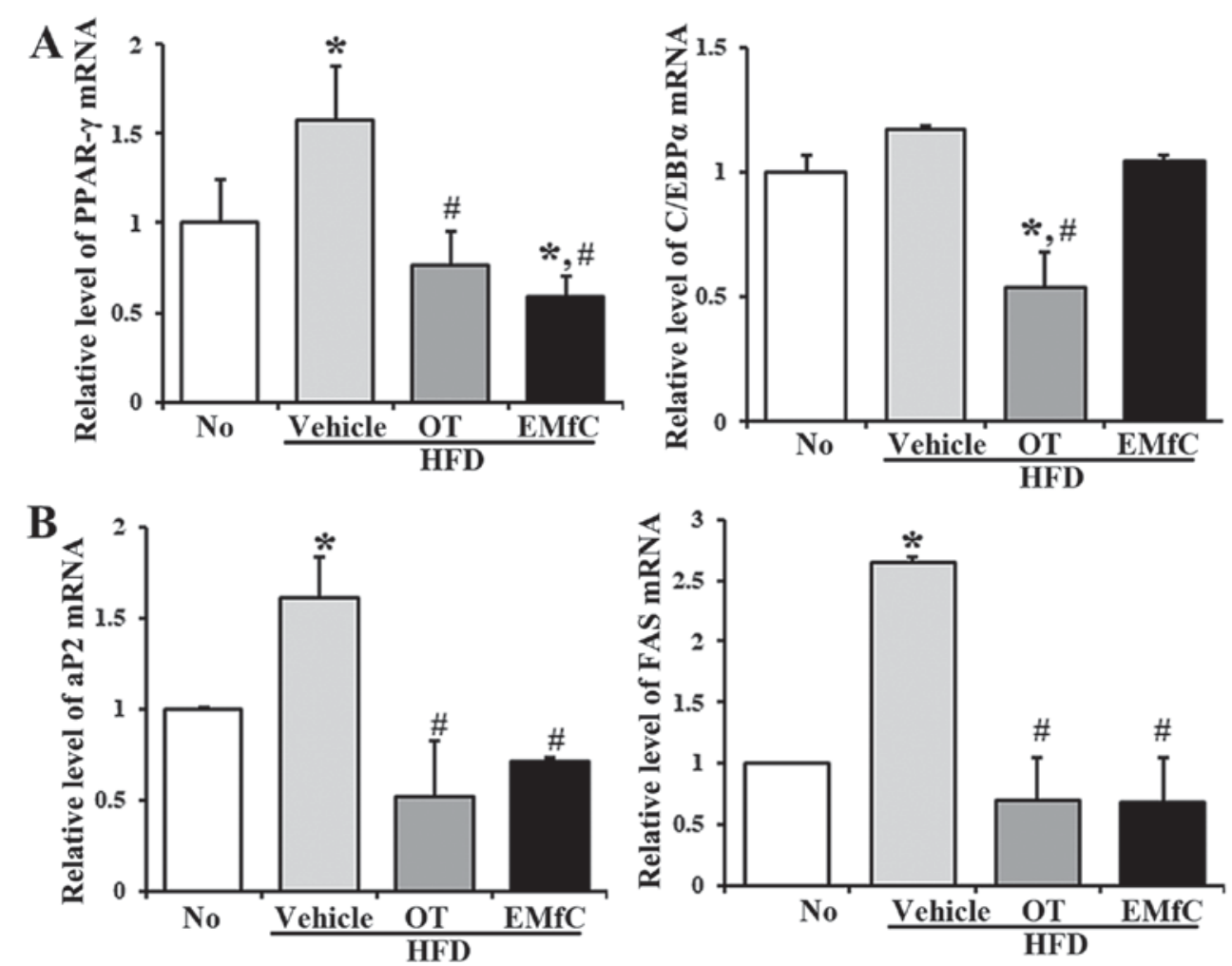

Figure 4. Expression of adipogenesis and lipogenesis associated genes in liver tissue. HFD-induced obesity C57BL/6 mice were treated with Vehicle, OT $(10 \mathrm{mg} / \mathrm{kg})$ or EMfC $(50 \mathrm{mg} / \mathrm{kg})$ for 12 weeks. Reverse transcription-quantitative polymerase chain reaction using specific primers measured the expression level of genes associated with (A) adipogenesis and (B) lipogenesis in the liver. The intensity of each band was determined using an imaging densitometer, and the relative levels of each gene were calculated based on the intensity of actin. Data is presented as the mean \pm standard deviation of the three experiments. ${ }^{*} \mathrm{P}<0.05$ vs. the No treatment group; ${ }^{*} \mathrm{P}<0.05$ vs. the HFD+Vehicle treated group. HFD, high fat diet; OT, Orlistat; EMfC, extract of mulberry leaves fermented with Cordyceps militaris; PPAR, peroxisome proliferator-activated receptor; C/EBP, CCAAT-enhancer binding protei; FAS, Fas cell surface death receptor; aP2, adipocyte protein 2 .
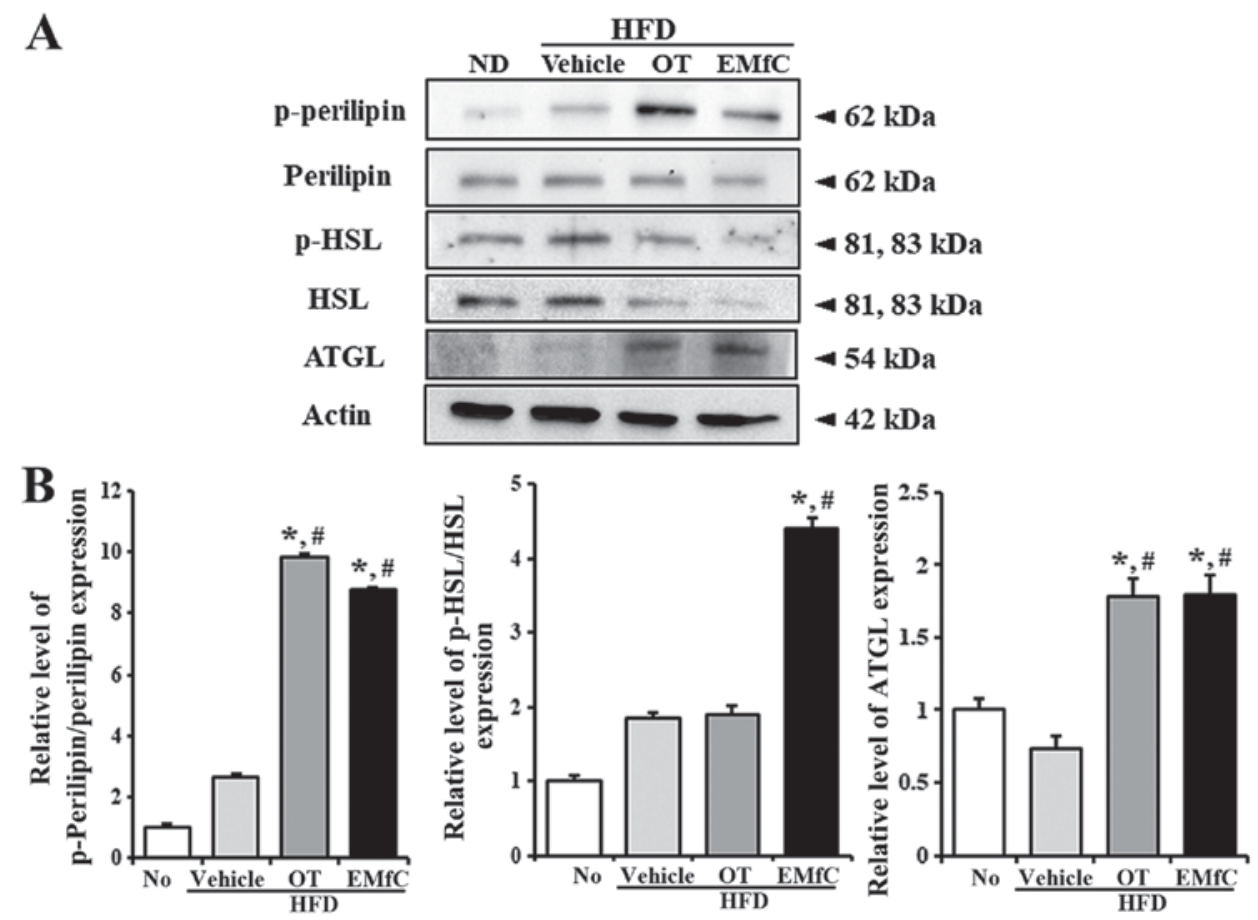

Figure 5. Expression of lipolysis associated proteins in liver tissues. (A) Western blot analysis measured the phosphorylation or expression of several lipolysis associated proteins, including (B) perilipin, p-perilipin, HSL, p-HSL and ATGL. The intensity of each band was determined using an imaging densitometer, and the relative levels of the four proteins were calculated based on the intensity of actin. Five to six rats per group were assayed in triplicate by western blotting. Data are presented as the mean \pm standard deviation of three replicates. ${ }^{*} \mathrm{P}<0.05$ vs. the No treatment group; ${ }^{*} \mathrm{P}<0.05$ vs. the HFD+Vehicle treated group. HFD, high fat diet; OT, Orlistat; EMfC, extract of mulberry leaves fermented with Cordyceps militaris; p-, phosphorylated; HSL, hormone-sensitive lipase; ATGL, adipose triglyceride lipase. 
adipocyte size and cell number, and it is therefore possible to control cardiovascular diseases by decreasing the concentration of LDL and TC in serum. Therefore, we believe that the association between cytokine and hormones involved in fat accumulation (such as adipokine and leptin), and the relationship with cardiovascular diseases should be studied further, with respect to the anti-obesity effects of EMfC.

Adipose tissue is responsible for systemic energy homeostasis. PPAR $\gamma$, a predominant transcription factor in adipose tissue, plays an important role in adipocyte differentiation, lipid storage, and glucose homeostasis $(37,38)$. PPAR $\gamma$ regulates the gene expression of enzymes involved in the lipogenesis of adipose tissue, such as FAS, aP2, and lipoprotein lipase $(39,40)$. FAS is a major lipogenic enzyme that catalyzes the biosynthesis of long chain fatty acids from acetyl-CoA precursors (41), and aP2 is an intracellular fatty acid binding protein that binds long-chain fatty acids (42). In this study, EMfC significantly reduced the mRNA levels of PPAR $\gamma, \mathrm{aP} 2$, and FAS in the HFD-induced obesity mice. These results suggest that EMfC treatment improves the intracellular fatty acid metabolism by inhibiting the lipogenic gene expression. Interestingly, there was no inhibition in the expression of C/EBP $\alpha$, a transcription factor similar to PPAR $\gamma$ and involved in lipogenesis, after EMfC administration. Orlistat inhibits the expression of two transcription factors (PPAR $\gamma$ and $\mathrm{C} / \mathrm{EBP} \alpha$ ) involved in lipid synthesis, suggesting that EMfC has a different mechanism for inhibiting lipid synthesis.

In addition to the synthesis of TG stored in adipose tissue, lipolysis (hydrolysis) is an important mechanism for fat accumulation in adipose tissue. ATGL (PNPLA2, desnutrin) is an important enzyme that initiates TG degradation and produces diacylglycerol (DG). It serves to initiate the sequential process of TG digestion, limiting the rate at the first step and determining the maximum rate for fatty acid mobilization (43-45). We observed that EMfC increases the amount of ATGL protein expression, similar to orlistat treatment. Perilipin is activated by the phosphorylation by phosphorylated PKA, which in turn activates the lipase of ATGL (46). Perilipin phosphorylation also increases after exposure to orlistat or EMfC, suggesting that EMfC has a similar lipid-breakdown mechanism to the orlistat induced mechanism. Interestingly, the phosphorylation of HSL, where ATGL degrades the DG into monoglycerides, occurs more strongly in EMfC than in orlistat (47), suggesting that EMfC has better activity in lipolysis than orlistat.

In conclusion, our study demonstrates that EMfC treatment successfully reduces the accumulation of adipose tissue and adipocyte cell size, and decrease the serum LDL, TG, TC and glucose levels in HFD-induced obese mice. In addition, there is a decrease in the liver weight and lipid drops, suppression in the expression of the lipogenic gene, and induction in the expression and activation of lipolysis protein. The strong effects of EMfC on fat loss and fatty liver loss without any accompanying adverse health effects suggests EMfC to be a potential anti-obesity drug that results in lipolysis.

\section{Acknowledgements}

The abstract was presented at the Korean Association for Laboratory Animal Science Winter Symposium Jan 24-Jan 27 2018 in Wonju, Kanwon-do, Korea and published as abstract no. OR-S-06 in Lab Anim Res 34 (Suppl. 1): 2018. The authors would like to thank their animal technician, Miss. Jin Hyang Hwang, for directing the animal care at the Laboratory Animal Resources Center in Pusan National University.

\section{Funding}

The present study was supported by grants from the Korea Institute of Planning \& Evaluation for Technology of Food, Agriculture and Forestry (grant no. 116027-032-HD030).

\section{Availability of data and materials}

The datasets used and/or analyzed during the current study are available from the corresponding author on reasonable request.

\section{Authors' contributions}

MRL, JEK, JYC, JJP, HRK, BRS, YWC, KMK and DYH participated in the design of the study, sample preparation, animal experiments and data analyses. HS performed the data analysis and assisted with manuscript preparation.

\section{Ethics approval and consent to participate}

The Pusan National University Animal Ethics Committee (no. PNU-2017-1519) approved the protocol for the present study.

\section{Patient consent for publication}

Not applicable.

\section{Competing interests}

The authors declare that they have no competing interests.

\section{References}

1. Haslam DW and James WP: Obesity. Lancet 366: 1197-1209, 2005.

2. Després JP and Lemieux I: Abdominal obesity and metabolic syndrome. Nature 444: 881-887, 2006.

3. NCD Risk Factor Collaboration (NCD-RisC): Worldwide trends in diabetes since 1980: A pooled analysis of 751 population-based studies with 4.4 million participants. Lancet 387: 1513-1530, 2016.

4. Maguire T and Haslam D: The Obesity Epidemic and its Management. Matthew Wright (ed) Pharmaceutical Press, London, pp264-267, 2010.

5. Pittler MH, Schmidt K and Ernst E: Adverse events of herbal food supplements for body weight reduction: Systematic review. Obes Rev 6: 93-111, 2005.

6. Gunjal S, Ankola AV and Bhat K: In vitro antibacterial activity of ethanolic extract of Morus alba leaf against periodontal pathogens. Indian J Dent Res 26: 533-536, 2015.

7. Raman ST, Ganeshan AK, Chen C, Jin C, Li SH, Chen HJ and Gui Z: In vitro and in vivo antioxidant activity of flavonoid extracted from mulberry fruit (Morus alba L.). Pharmacogn Mag 12: 128-133, 2016.

8. Wang Y, Xiang L, Wang C, Tang C and He X: Antidiabetic and antioxidant effects and phytochemicals of mulberry fruit (Morus alba L.) polyphenol enhanced extract. PLoS One 8: e71144, 2008.

9. Jo SP, Kim JK and Lim YH: Antihyperlipidemic effects of stilbenoids isolated from Morus alba in rats fed a high-cholesterol diet. Food Chem Toxicol 65: 213-218, 2014. 
10. El-Beshbishy HA, Singab AN, Sinkkonen J and Pihlaja K: Hypolipidemic and antioxidant effects of Morus alba L. (Egyptian mulberry) root bark fractions supplementation in cholesterol-fed rats. Life Sci 78: 2724-2733, 2006.

11. Eo HJ, Park JH, Park GH, Lee MH, Lee JR, Koo JS and Jeong JB Anti-inflammatory and anti-cancer activity of mulberry (Morus alba L.) root bark. BMC Complement Altern Med 14: 200, 2014

12. Chan EW, Lye PY and Wong SK: Phytochemistry, pharmacology, and clinical trials of Morus alba. Chin J Nat Med 14: 17-30, 2016.

13. Kobayashi Y, Miyazawa M, Kamei A, Abe K and Kojima T: Ameliorative effects of mulberry (Morus alba L.) leaves on hyperlipidemia in rats fed a high-fat diet: Induction of fatty acid oxidation, inhibition of lipogenesis, and suppression of oxidative stress. Biosci Biotechnol Biochem 74: 2385-2395, 2010.

14. Yang SJ, Park NY and Lim Y: Anti-adipogenic effect of mulberry leaf ethanol extract in 3T3-L1 adipocytes. Nutr Res Pract 8: 613-617, 2014.

15. Ann JY, Eo HY and Lim YS: Mulberry leaves (Morus alba L.) ameliorate obesity-induced hepatic lipogenesis, fibrosis, and oxidative stress in high-fat diet-fed mice. Genes Nutr 10: 46, 2015.

16. Naowaboot J, Pannangpetch P, Kukongviriyapan V, Prawan A, Kukongviriyapan U and Itharat A: Mulberry leaf extract stimulates glucose uptake and GLUT4 translocation in rat adipocytes. Am J Chin Med 40: 163-175, 2012.

17. Sugimoto M, Arai H, Taura Y, Murayama T, Khaengkhan $P$, Nishio T, Ono K, Ariyasu H, Akamizu T, Ueda Y, et al: Mulberry leaf ameliorates the expression profile of adipocytokines by inhibiting oxidative stress in white adipose tissue in $\mathrm{db} / \mathrm{db}$ mice. Atherosclerosis 204: 388-394, 2009.

18. Sung GH, Hywel-Jones NL, Sung JM, Luangsa-Ard JJ, Shrestha B and Spatafora JW: Phylogenetic classification of Cordyceps and the clavicipitaceous fungi. Stud Mycol 57: 5-59, 2007.

19. De Silva DD, Rapior S, Sudarman E, Stadler M, Xu J, Alias SA and Hyde KD: Bioactive metabolites from macrofungi: Ethnopharmacology, biological activities and chemistry. Fungal Divers 62: 1-40, 2013.

20. De Silva DD, Rapior S, Fons F, Bahkali AH and Hyde KD: Medicinal mushrooms in supportive cancer therapies: An approach to anti-cancer effects and putative mechanisms of action. Fungal Divers 55: 1-35, 2012.

21. Pao HY, Pan BS, Leu SF and Huang BM: Cordycepin stimulated steroidogenesis in MA-10 mouse Leydig tumor cells through the protein kinase C Pathway. J Agric Food Chem 60: 4905-4913, 2102.

22. Kim JR, Yeon SH, Kim HS and Ahn YJ: Larvicidal activity against Plutella xylostella of cordycepin from the fruiting body of Cordyceps militaris. Pest Manag Sci 58: 713-717, 2012.

23. Ramesh T, Yoo SK, Kim SW, Hwang SY, Sohn SH, Kim IW and Kim SK: Cordycepin (3'-deoxyadenosine) attenuates age-related oxidative stress and ameliorates antioxidant capacity in rats. Exp Gerontol 47: 979-987, 2012.

24. Guo P, Kai Q, Gao J, Lian ZQ, Wu CM, Wu CA and Zhu HB: Cordycepin prevents hyperlipidemia in hamsters fed a high-fat diet via activation of AMP-activated protein kinase. J Pharm Sci 113: 395-403, 2010.

25. Kim SS, Lim KS, Kim HJ, Chong MS, Cho HE, Choi YH, et al: Effects of extracts from mixed culture with Tricholoma Matsutake mycelium and Cordyceps Militaris mycelium on mlood glucose in streptozotocin-induced diabetic rats. Korean J Orient Physiol Pathol 22: 365-370, 2008.

26. Hung YP and Lee CL: Higher anti-liver fibrosis effect of cordyceps militaris-fermented product cultured with deep ocean water via inhibiting proinflammatory factors and fibrosis-related factors expressions. Mar Drugs 15: pii: E168, 2017.

27. Lee MR, Kim JE, Yun WB, Choi JY, Park JJ, Kim HR, Song BR, Choi YW, Kim KM and Hwang DY: Lipolytic effect of novel extracts from mulberry (Morus alba) leaves fermented with Cordyceps militaris in the primary adipocytes derived from SD rats. Lab Anim Res 33: 270-279, 2017.
28. Livak KJ and Schmittgen TD: Analysis of relative gene expression data using real-time quantitative PCR and the 2(-Delta Delta C(T)) method. Methods 25: 402-408, 2001

29. Hofbauer KG, Nicholson JR and Boss O: The obesity epidemic: Current and future pharmacological treatments. Annu Rev Pharmacol Toxicol 47: 565-592, 2007.

30. Sharma B and Henderson DC: Sibutramine: Current status as an anti-obesity drug and its future perspectives. Expert Opin Pharmacother 9: 2161-2173, 2008.

31. Lean ME: Pathophysiology of obesity. Proc Nutr Soc 59: 331-336, 2000.

32. Apostolopoulou M, Savopoulos C, Michalakis K, Coppack S, Dardavessis T and Hatzitolios A: Age, weight and obesity. Maturitas 71: 115-119, 2012.

33. Morimoto C, Kameda K, Tsujita T and Okuda H: Relationships between lipolysis induced by various lipolytic agents and hormone-sensitive lipase in rat fat cells. J Lipid Res 42: 120-127, 2001.

34. Bray GA: Drug treatment of obesity. Rev Endocr Metab Disord 2: 403-418, 2001.

35. Mayer MA, Höcht C, Puyó A and Taira CA: Recent advances in obesity pharmacotherapy. Curr Clin Pharmacol 4: 53-61, 2009.

36. Vázquez-Vela ME, Torres N and Tovar AR: White adipose tissue as endocrine organ and its role in obesity. Arch Med Res 39: 715-728, 2008

37. Schoonjans K, Staels B and Auwerx J: The peroxisome proliferator activated receptors (PPARS) and their effects on lipid metabolism and adipocyte differentiation. Biochim Biophys Acta 1302: 93-109, 1996.

38. Rosen ED, Walkey CJ, Puigserver P and Spiegelman BM: Transcriptional regulation of adipogenesis. Genes Dev 14: 1293-1307, 2000.

39. Kliewer SA and Willson TM: The nuclear receptor PPARgamma-bigger than fat. Curr Opin Genet Dev 8: 576-581, 1998.

40. Spiegelman BM: PPAR-gamma: Adipogenic regulator and thiazolidinedione receptor. Diabetes 47: 507-514, 1998.

41. Boizard M, Le Liepvre X, Lemarchand P, Foufelle F, Ferré P and Dugail I: Obesity-related overexpression of fatty-acid synthase gene in adipose tissue involves sterol regulatory element-binding protein transcription factors. J Biol Chem 273: 29164-29171, 1998.

42. Coe NR and Bernlohr DA: Physiological properties and functions of intracellular fatty acid-binding proteins. Biochim Biophys Acta 1391: 287-306, 1998.

43. Zimmermann R, Strauss JG, Haemmerle G, Schoiswohl G, Birner-Gruenberger R, Riederer M, Lass A, Neuberger G, Eisenhaber F, Hermetter A and Zechner R: Fat mobilization in adipose tissue is promoted by adipose triglyceride lipase. Science 306: 1383-1386, 2004.

44. Haemmerle G, Lass A, Zimmermann R, Gorkiewicz G, Meyer C, Rozman J, Heldmaier G, Maier R, Theussl C, Eder S, et al: Defective lipolysis and altered energy metabolism in mice lacking adipose triglyceride lipase. Science 312: 734-737, 2006.

45. Ahmadian M, Duncan RE, Varady KA, Frasson D, Hellerstein MK, Birkenfeld AL, Samuel VT, Shulman GI, Wang Y, Kang C and Sul HS: Adipose overexpression of desnutrin promotes fatty acid use and attenuates diet-induced obesity. Diabetes 58: 855-866, 2009.

46. Tansey JT, Sztalryd C, Hlavin EM, Kimmel AR and Londos C: The central role of perilipin a in lipid metabolism and adipocyte lipolysis. IUBMB Life 56: 379-385, 2004.

47. Marcelin G and Chua S Jr: Contributions of adipocyte lipid metabolism to body fat content and implications for the treatment of obesity. Curr Opin Pharmacol 10: 588-593, 2010.

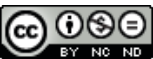

This work is licensed under a Creative Commons Attribution-NonCommercial-NoDerivatives 4.0 International (CC BY-NC-ND 4.0) License. 